\title{
Minimum cost design of a welded stiffened square plate loaded by biaxial compression
}

\author{
József Farkas*, Luis M.C. Simões** and Károly Jármai* \\ * University of Miskolc, H-3515 Hungary, altfar@gold.uni-miskolc.hu, altjar@gold.uni-miskolc.hu \\ ** University of Coimbra, Portugal, lcsimoes@alfa.dec.uc.pt
}

\begin{abstract}
1. Abstract
The optimized plate structure consists of a simply supported square base plate stiffened with an orthogonal grid of flat stiffeners welded to the base plate by fillet welds. The uniformly distributed compressive load is acting biaxially in the plane determined by the gravity centers of T-sections, which consist of a part of base plate and of a stiffener. In the optimization process the number of stiffeners as well as the thicknesses of base plate and flat stiffeners are sought, which minimize the cost function and fulfil the design constraints. The cost function includes the cost of material, assembly, welding and painting. Constraints relate to the global buckling, local buckling of base plate parts and stiffeners as well as to the deflection due to shrinkage of welds. To illustrate the effectiveness of mathematical methods, the problem is solved by the Rosenbrock s hillclimb algorithm as well as by entropy-based unconstrained minimization. Keywords: minimum cost design, structural optimization, buckling strength, stiffened plates, welded structures
\end{abstract}

\section{Introduction}

Our aim is to show the application of efficient mathematical methods to an important optimization problem. Stiffened plates are used as load-carrying elements of ships, bridges, offshore platforms, roofs, etc. In stability problems of welded structures the effect of initial imperfections and residual welding stresses should be taken into account. Based on own experimental results Mikami and Niwa [1] have proposed formulae for the calculation of ultimate buckling strength of orthogonally stiffened plates loaded by uniaxial compression considering the above mentioned effects. Their method is used in an article of Farkas and Jármai [2] and extended here for plates compressed biaxially.

For the objective function an advanced cost function is used including material, welding and painting costs. Fabrication cost plays an important role in the whole cost and the comparison of the minimum costs of different structural versions enables designers to achieve significant cost savings in the design stage.

To illustrate the effectiveness of the mathematical methods, the problem is solved by using the Rosenbrock s Hillclimb method [3], and by an entropy-based unconstrained minimization for the optimization of continuous design variables associated with a branch and bound strategy [4].

\section{Problem formulation}

In an effective structural optimization the variables are selected on the basis of analysis of structural characteristics. In the case of stiffened plates the structural characteristics are as follows.

Loads: uniaxial or biaxial compression, in-plane bending and shear (plate girder webs), lateral pressure, hydrostatic pressure, concentrated, distributed on a line, uniformly distributed, static, dynamic, variable, high temperature.

Material: normal or high-strength steels, aluminium-alloys.

Plate geometry: square, rectangular, triangular, trapezoidal, circular.

Boundary conditions: simply supported, clamped, free, elastic support.

Stiffening geometry: edge-parallel, diagonal-parallel, unidirectional, orthogonal, tridirectional, circular.

Topology: number of stiffeners (variable).

Stiffener shape: flat, rolled T- and L-profile, welded T-profile, cold-formed L-profile, trapezoidal, rectangular hollow section. Possible variables: dimensions of stiffeners.

Connections of stiffeners to base plate: welded, riveted, bolted or bonded.

Fabrication of nodes: welded, riveted, bolted or bonded with L-elements.

From these characteristics we have selected for this study the following.

The investigated plate structure (Figure 1) consists of a simply supported square base plate stiffened with an orthogonal grid of flat stiffeners welded to the base plate by fillet welds. It is assumed that the stiffeners in one direction are continuous and in the other direction they are intermittent. The connections of stiffeners are welded by transverse fillet welds.

The uniformly distributed compressive load is acting biaxially in the plane determined by the gravity centers of Tsections, which consist of a part of the base plate and of a stiffener.

The unknown variables are as follows. $\boldsymbol{\varphi}=b / a ; t_{F} ; h_{S} ; t_{S} . b$ is the whole side length of the base plate, $t_{F}$ is the thickness of the base plate, $h_{S}$ and $t_{S}$ are the height and the thickness of a flat stiffener. Thus, the number of stiffeners in one direction is $\varphi-1$. The optima of the variables are sought, which minimize the cost function and fulfil the design constraints.

Numerical data: $b=8 \mathrm{~m} ; N=9800 \mathrm{kN}$; the yield stress is $f_{y}=235 \mathrm{MPa}$. 


\section{Cost function}

According to Farkas and Jármai $[3,5]$ the cost function includes material, fabrication (welding) and painting costs

$$
K=k_{M} \rho_{0} V+k_{F}\left[\Theta\left(\boldsymbol{p}_{0} V\right)^{1 / 2}+1.3\left(T_{2}^{\prime}+T_{2}^{\prime \prime}+T_{2}^{\prime \prime \prime}\right)\right]+k_{P} S
$$

The cost factors are as follows:

Material cost factor is $k_{M}=0.5-1.0 \$ / \mathrm{kg}$, fabrication cost factor is $k_{F}=12-48 \$ / \mathrm{h}=0.2-0.8 \$ / \mathrm{min}$, painting cost factor is $k_{P}=15 \$ / \mathrm{m}^{2}$. We calculate with $k_{M}=0.5 \$ / \mathrm{kg}$ and $k_{F}=0.6 \$ / \mathrm{min}$.

The density of the steel is $\rho_{0}=7.85 \times 10^{-6} \mathrm{~kg} / \mathrm{mm}^{3}$

The volume of the structure is

$$
V=b^{2} t_{F}+2 b(\varphi-1) h_{S} t_{S}
$$

the difficulty factor expressing the complexity of the structure is $\Theta=3$

the number of structural parts to be assembled is

$$
\kappa=16+\varphi-1+\varphi(\varphi-1)=17+\varphi^{2}
$$

Welding times are as follows

(a) butt welds of the base plate, in the numerical example the base plate side length is $b=8 \mathrm{~m}$, and it is assumed that this base plate is welded from plate elements of dimensions $6 \mathrm{mx} 1.5 \mathrm{~m}$, so the weld length is $L_{W}=8 b=64 \mathrm{~m}$, weld size is $t_{F}$, welding technology is GMAW-M (Gas metal arc welding with mixed gas)

$$
\begin{array}{lc}
\text { for } t_{F} \leq 15 \mathrm{~mm} & T_{2}^{\prime}=0.1861 t_{F}^{2} \times 64 \\
\text { for } t_{F}>15 \mathrm{~mm} & T_{2}^{\prime}=0.1433 t_{F}^{1.9035} \times 64
\end{array}
$$

(b) longitudinal fillet welds connecting the flat stiffeners to the base plate, welding technology is GMAW-M,

$$
T_{2}^{\prime \prime}=0.3258 \times 10^{-3} a_{W}^{2} x 4 b(\varphi-1)
$$

$a_{W}=0.4 t_{S}$, but $a_{W \cdot \min }=4 \mathrm{~mm}$

(c) transversal fillet welds connecting the intermittent flat stiffeners to the continuous ones.

Number of nodes is $(\varphi-1)^{2}$, welding technology is SMAW (Shielded metal arc welding)

The superficies to be painted is

$$
T_{2}^{\prime \prime \prime}=0.7889 \times 10^{-3} a_{W}^{2} x 4 h_{S}(\varphi-1)^{2}
$$

$$
S=2 b^{2}+4 b(\varphi-1) h_{S}
$$

\section{Design constraints}

5.1 Constraint on global buckling

$$
\frac{N}{A} \leq \sigma_{U}^{*}=\sigma_{U} \frac{\rho_{P}+\delta_{S}}{1+\delta_{S}}
$$

where $N$ is the compression force,

$$
A=b t_{F}+(\varphi-1) A_{S}
$$

$b=\varphi a$ is the side length of the whole plate, $a$ is the distance between stiffeners, $t_{F}$ is the thickness of the base plate,

$$
\delta_{S}=\frac{A_{S}}{a t_{F}}
$$

$\sigma_{U}$ is the ultimate global buckling strength of the whole, simply supported plate. It is calculated on the basis of the classic formula [6]

$$
\begin{gathered}
\sigma_{c r}=\frac{\pi^{2} D_{1}}{h b^{2}} \\
h=t_{F}+A_{S} / a \\
D_{1}=D+E I_{X} / a \\
D=\frac{E t_{F}^{3}}{12\left(1-v^{2}\right)}=\frac{E t_{F}^{3}}{10.92}
\end{gathered}
$$

$I_{X}$ is the moment of inertia of a cross-section containing the flat stiffener and a strip of the base plate of the width of $a$. The distance of the gravity center of this T-section is

$$
\begin{gathered}
y_{G}=\frac{h_{S}+t_{F}}{2} \frac{\delta_{S}}{1+\delta_{S}} \\
I_{X}=\frac{h_{S}^{3} t_{S}}{12} \frac{4+\delta_{S}}{1+\delta_{S}}
\end{gathered}
$$




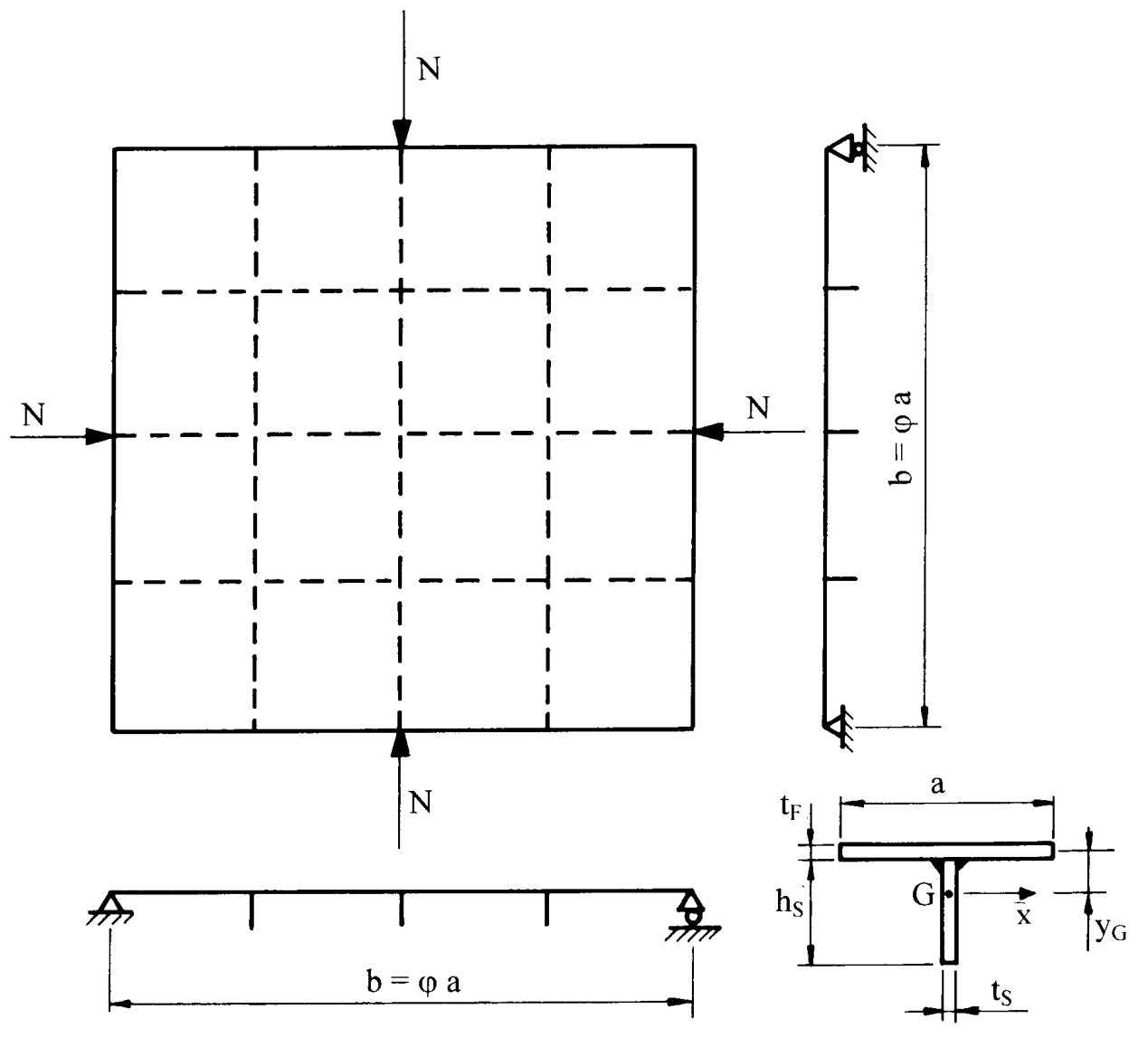

Figure 1. Welded square plate with flat stiffeners

$A_{S}=h_{S} t_{S}$ is the cross-sectional area of a flat stiffener, $h_{S}$ is the height and $t_{S}$ is the thickness of a flat stiffener,

Since the classic buckling strength formula does not take into account the effect of the initial imperfection and residual welding stresses, we use a reduced buckling strength according to Mikami and Niwa method based on a reduced slenderness

$$
\lambda_{R}=\left(f_{y} / \sigma_{c r}\right)^{1 / 2}
$$

where $f_{v}$ is the yield stress. Note that the method of this reduced slenderness is used also in Eurocode 3 [7]

$$
\begin{gathered}
\sigma_{U} / f_{y}=1 \quad \text { for } \quad \lambda_{R} \leq 0.3 \\
\sigma_{U} / f_{y}=1-0.63\left(\lambda_{R}-0.3\right) \quad \text { for } 0.3<\lambda_{R} \leq 1 \\
\sigma_{U} / f_{y}=1 /\left(0.8+\lambda_{R}^{2}\right) \quad \text { for } \lambda_{R}>1
\end{gathered}
$$

The factor of $\rho_{P}$ is introduced in order to take into consideration the local buckling of the base plate

$$
\begin{array}{cll}
\rho_{P}=1 \quad \text { if } & \sigma_{U P} \geq \sigma_{U} \\
\rho_{P}=\sigma_{U P} / f_{y} & \text { if } \sigma_{U P}<\sigma_{U}
\end{array}
$$

$\sigma_{U P}$ is the ultimate local buckling strength of a base plate square field, which is assumed to be simply supported and loaded by biaxial compression. The ultimate strength is calculated also by using a reduced slenderness derived from the classic buckling strength

$$
\begin{gathered}
\frac{\sigma_{P c r}}{f_{y}}=\frac{2 \pi^{2} E}{10.92 f_{y}}\left(\frac{t_{F}}{a}\right)^{2} \\
\lambda_{P}=\left(\frac{f_{y}}{\sigma_{P c r}}\right)^{1 / 2}=\frac{a / t_{F}}{40.19 \varepsilon} \quad \varepsilon=\left(235 / f_{y}\right)^{1 / 2}
\end{gathered}
$$

$f_{y}$ is the actual yield stress in MPa.

$$
\frac{\sigma_{U P}}{f_{y}}=1 \quad \text { for } \quad \lambda_{P} \leq 0.526
$$




$$
\frac{\sigma_{U P}}{f_{y}}=\left(\frac{0.526}{\lambda_{P}}\right)^{0.7} \quad \text { for } \quad \lambda_{P} \geq 0.526
$$

5.2 Constraint on local buckling of flat stiffeners

According to Eurocode 3 (1992)

$$
h_{S} / t_{S} \leq 14 \varepsilon
$$

5.3 Distortion constraint

In order to assure the quality of this type of welded structures large deflections due to weld shrinkage should be avoided. It has been shown that the curvature of a beam like structure due to shrinkage of longitudinal and transverse welds can be calculated by relatively simple formulae [3]. The allowable maximal residual deformation $f_{0}$ is prescribed by design rules. For compression Eurocode 3 [7] prescribes $f_{0}=b / 1000$, thus the distortion constraint is defined as

where the curvature for steel is

$$
f_{\max }=1.5 \quad C b^{2} / 8 \leq f_{0}=b / 1000
$$

$Q_{T}$ is the heat input, $y_{T}$ is the weld eccentricity

$$
C=0.844 \times 10^{-3} \quad Q_{T} y_{T} / I_{x}
$$

$y_{G}$ and $I_{x}$ are given by Eq.(16) and Eq.(17).

$$
\mathrm{y}_{\mathrm{T}}=\mathrm{y}_{\mathrm{G}}-\mathrm{t}_{\mathrm{F}} / 2
$$

5.4 Limitation of the number of spacings between the stiffeners

Since the classic overall buckling strength is calculated on the basis of the theory of orthotropic plates, a limitation of the number of spacings

$$
\varphi \geq 3
$$

should be introduced.

\section{The applied mathematical methods}

6.1 Optimum design with continuous design variables

For solving each relaxed problem with continuous design variables the simultaneous minimization of the cost and constraints is sought. All these goals are cast in a normalized form. If a reference cost $\mathrm{K}_{0}$ is specified, this goal can be written in the form,

$$
\mathrm{g}_{1}\left(t_{F}, t_{s}\right)=\mathrm{K}\left(t_{F}, t_{s}\right) / \mathrm{K}_{\mathrm{o}}-1 \leq 0
$$

A second goal arises from the constraint on overall buckling:

$$
\mathrm{g}_{2}\left(t_{F}, t_{s}\right)=N(1+\delta) /\left(\rho_{p}+\delta\right) A \sigma_{U}-1 \leq 0
$$

The remaining goal deals with the limitations of distortions:

$$
\mathrm{g}_{3}\left(t_{F}, t_{s}\right)=1500 C b / 8-1 \leq 0
$$

The objective of this Pareto optimization is to obtain an unbiased improvement of the current design which can be found by the unconstrained minimization of the convex scalar function:

$$
\mathrm{F}\left(t_{F}, t_{S}\right)=\frac{1}{\rho} \cdot \ln \left[\sum_{\mathrm{j}=1}^{3} \exp \rho\left(g\left(t_{F}, t_{S}\right)\right)\right]
$$

This form leads to a convex conservative approximation to the objective and constraint boundaries. Accuracy increases with $\rho$.

The strategy adopted was an iterative sequence of explicit approximation models, formulated by taking Taylor series approximations of all the goals truncated after the linear term. This gives:

$$
\operatorname{Min} \mathrm{F}\left(t_{F}, t_{S}\right)=\frac{1}{\rho} \cdot \ln \left[\sum_{\mathrm{j}=1}^{3} \exp \rho\left(g_{0}\left(t_{F}, t_{S}\right)+\frac{\partial g_{0 j}\left(t_{F}, t_{S}\right)}{\partial t_{F}} d t_{F}+\frac{\partial g_{0 j}\left(t_{F}, t_{S}\right)}{\partial t_{S}} d t_{S}\right)\right]
$$


This problem has an analytic solution giving the design variables changes $\mathrm{d} t_{F}$ and $\mathrm{d} t_{s}$. Solving for a particular numerical value of $g_{\mathrm{oj}}$ forms an iteration of the solution to problem Eq.(36). Move limits must be imposed on the design variable changes to guarantee the accuracy of the approximations. The new design must be analysed giving the values for the cost function and the other goals. New solutions to Eq.(37) are obtained until design variable changes become small. During the iterations the control parameter $\rho$, which should not be decreased to produce an improved solution, is increased from 30 to 200 .

Other alternative procedures could be efficiently used to locate optimum solutions with continuous design variables. Caution is required given the nonconvexity of the total cost function and the constraints for $14 \mathrm{~mm} \leq t_{F} \leq 16 \mathrm{~mm}$.

\subsection{Branch and Bound}

The problem was a non-linear objective function and domain. Moreover the solution procedures which can be adopted to handle this type of problems can be characterized as deterministic (enumerative strategies, cutting planes, tunnelling methods), stochastic (random search, simulated annealing) or based on analogies with biology (genetic programming, evolutionary method). Given the small number of discrete design variables an implicit branch and bound strategy was adopted to find the least cost solution.

The two main ingredients are a combinatorial tree with appropriately defined nodes and some upper and low bounds to the optimum solution associated the nodes of the tree. It is then possible to eliminate a large number of potential solutions without evaluating them. As the implicit numeration relies on the upper bound, its efficiency can be greatly improved by providing a good feasible initial solution.

A partial solution is said to be fathomed if the best completion of the solution can be found or or if it can be determined that, no matter how sections are assigned to the remaining free members it will be impossible to find a feasible completion of smaller cost than the previously found. If a partial solution is fathomed this means that all possible completions of the partial solution have been implicitly enumerated. When the last node is fathomed the algorithm ends up with the optimum design. Backtracking in the tree is performed so that no solution is repeated or omitted from consideration. An underestimate of the optimum solution can be found if the discrete design variables requirement is relaxed. The number of levels in the combinatorial tree equals the number of discrete design variables.

Each node can be branched into $\mathrm{n}$ new nodes where $\mathrm{n}$ represents the set of available sections of the selected design variable. The combinatorial tree up to level $n-1$ has each node identified with an underestimate. Each node of the tree is associated with an incumbent bound. Any leaf of the tree whose bound is strictly less than the incumbent is active. Otherwise it is designated as terminated and need not to be considered further. The B\&B tree is developed until every leaf is terminated (Figure 2). The branching strategy adopted was breadth first, consisting of choosing the node with the lower bound.

\subsection{The method of Rosenbrock}

The second algorithm applied is Rosenbrock's method, which has also been modified to be able to handle discrete values [3]. This method is a direct search mathematical programming method without derivatives. Instead of continuous line searches, the algorithm takes discrete steps during searches in orthogonal search directions. In each iteration, the procedure searches successively along $n$ linearly independent and orthogonal directions. When a new point is reached at the end of an iteration, a new set of orthogonal search vectors are constructed. Boundary zones are introduced to slow down the algorithm when it approaches the constraint boundaries. A modified objective function, using penalty functions, are used to handle the constraints. Instead of continually searching in the co-ordinate space corresponding to the directions of the independent variables, the method achieves an improvement after one cycle of co-ordinate searches by lining the search directions up into an orthogonal system, with the overall step of the previous stage as the first building block for the new set of orthogonal directions. After each iteration $k$, Rosenbrock's method locates $\mathbf{x}^{(k+1)}$ after completing unidimensional searches from the previous point $\mathbf{x}^{(k)}$ along a set of orthonormal directions. It introduces boundary zones, to slow down the algorithm, when it approaches the boundary too closely. A modified objective function is calculated in the boundary zone, using penalty functions. No gradient calculation is needed. The available computer code is very easy to implement on engineering problems. The method may find local minima instead of the global minimum.

\section{Results and conclusions}

The results are given in Tables 1-4. Minimum material cost and minimum total cost results for continuous design variables can be found in Tables 1,2. Optimum discrete design variables are given in Tables 3,4.

The optima are marked by bold letters.

It can be seen that the optimum number of spacings for minimum material cost differs from that for minimum whole cost. The optimal number of spacings (stiffeners) is smaller for minimum whole cost. This decrease is caused by high fabrication costs. The cost differences between the best and worst structural solutions indicated in tables are $18-22 \%$, 
thus, it is worth using the optimization procedure. Both mathematical optimization methods have been efficient for this problem.

Table 1. Minimum material cost data continuous design variables

\begin{tabular}{cccc}
\hline $\boldsymbol{\varphi}$ & $t_{f}$ & $T_{S}$ & $K_{M} \$$ \\
\hline 3 & 18.7 & 19.8 & 5362 \\
4 & 16.2 & 18.8 & 5000 \\
$\mathbf{5}$ & $\mathbf{1 4 . 5}$ & $\mathbf{1 8 . 0}$ & $\mathbf{4 7 7 4}$ \\
6 & 14.6 & 17.1 & 4951 \\
7 & 15.5 & 16.4 & 5310 \\
\hline
\end{tabular}

Table 2. Minimum total cost data continuous design variables

\begin{tabular}{cccc}
\hline $\boldsymbol{\varphi}$ & $t_{f}$ & $t_{S}$ & $K \$$ \\
\hline 3 & 18.7 & 19.8 & 11603 \\
$\mathbf{4}$ & $\mathbf{1 6 . 1}$ & $\mathbf{1 8 . 7}$ & $\mathbf{1 1 4 2 3}$ \\
5 & 15.0 & 17.9 & 12714 \\
6 & 15.0 & 17.1 & 13847 \\
7 & 15.5 & 16.4 & 13695 \\
\hline
\end{tabular}

4774

$14.49 ; 17.96$

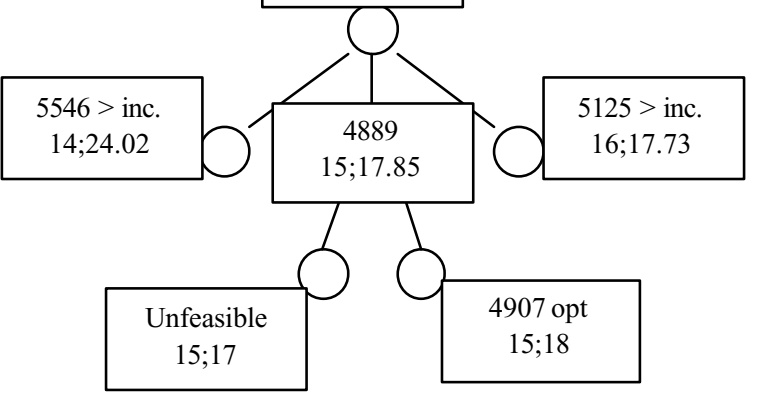

Figure 2. Branch and Bound Tree
Table 3. Minimum material cost data discrete design variables

\begin{tabular}{cccc}
\hline $\boldsymbol{\varphi}$ & $t_{f}$ & $t_{S}$ & $K_{M} \$$ \\
\hline 3 & 19 & 20 & 5476 \\
4 & 17 & 19 & 5222 \\
$\mathbf{5}$ & $\mathbf{1 5}$ & $\mathbf{1 8}$ & $\mathbf{4 9 0 7}$ \\
6 & 16 & 17 & 5289 \\
7 & 17 & 17 & 5795 \\
\hline
\end{tabular}

Table 4. Minimum total cost data discrete design variables

\begin{tabular}{cccc}
\hline $\boldsymbol{\varphi}$ & $t_{f}$ & $t_{S}$ & $K \$$ \\
\hline $\mathbf{3}$ & $\mathbf{1 9}$ & $\mathbf{2 0}$ & $\mathbf{1 1 7 8 7}$ \\
4 & 17 & 19 & 11905 \\
5 & 16 & 18 & 12349 \\
6 & 16 & 17 & 13087 \\
7 & 17 & 17 & 14779 \\
\hline
\end{tabular}

When $\boldsymbol{\varphi}$ is increased, $t_{S}$ becomes smaller to reduce distortion. $t_{f}$ is reduced with $\boldsymbol{\varphi}$, but for larger $\boldsymbol{\varphi}$ it must compensate the diminishing $t_{S}$.

\section{Acknowledgements}

The research work was supported by the Hungarian Scientific Research Found grants OTKA 22846, 29326, Fund for the Development of Higher Education FKFP 8/2000 project, the Portuguese Institute for Scientific and Technological Cooperation ICCTI and Oriente Foundation.

\section{References}

1. Mikami I and Niwa K. Ultimate compressive strength of orthogonally stiffened steel plates. J. Struct. Eng. ASCE, 1996, 122(6):674-682. Discussion and closure J.Struct.Eng. ASCE, 1997, 123(8):1116-1119

2. Farkas $\mathrm{J}$ and Jármai K. Minimum cost design and comparison of uniaxially compressed plates with welded flat, Land trapezoidal stiffeners. Welding in the World, 2000, 44(3):47-51

3. Farkas J and Jármai K. Analysis and optimum design of metal structures. Rotterdam-Brookfield: Balkema, 1997

4. Simoes LM C and Negrao J H J O. Optimization of cable-stayed bridges subjected to earthquakes with nonlinear behaviour. Eng. Opt. 1999, 31:457-478

5. Jármai K and Farkas J. Cost calculation and optimization of welded steel structures. J. Constructional Steel Research, 1999, 50(2):115-135

6. American Petroleum Institute. API Bulletin on design of flat plate structures. Bul.2V. 1987

7. Eurocode 3. Design of steel structures. Part 1.1. General rules and rules for buildings. Brussels, 1992 